\title{
Additive Effect of ANRIL and BRAP Polymorphisms on Ankle-Brachial Index in a Taiwanese Population
}

\author{
Pei-Chien Tsai; Yi-Chu Liao; Tsung-Hsien Lin; Edward Hsi; \\ Yi-Hsin Yang; Suh-Hang Hank Juo
}

\begin{abstract}
Background: Genetic variations on chromosome 9p21 have been found to be associated with peripheral artery disease (PAD), but have not been investigated in Asians. The ankle-brachial index ( $A B I)$ is a widely-used measurement for PAD. We previously reported the BRAP gene is associated with the $A B I$, so the aim of the present study was to test whether the $A N R I L$ gene on $9 \mathrm{p} 21$ is associated with the $\mathrm{ABI}$ and to test an interaction between $B R A P$ and $A N R I L$ in a Chinese population.
\end{abstract}

\begin{abstract}
Methods and Results: A total of 745 subjects with a family history of myocardial infarction or stroke were enrolled. The multiplicative and additive effects of 2 significant single-nucleotide polymorphisms (SNPs) were evaluated by multivariable regression analysis. SNP rs2383207 on ANRIL was most significantly associated with lower ABI. Similar to our previous findings, SNP rs11066001 on BRAP was associated with lower ABI. A dose-response relationship between $A B I$ values and the number of risk alleles from the 2 significant SNPs was observed in both men and women (adjusted $\mathrm{P}=0.004$ for men, 0.008 for women). The combined genetic effect on $\mathrm{ABI}$ was stronger in smokers than in non-smokers.
\end{abstract}

Conclusions: ANRIL on 9p21 and BRAP were both associated with $\mathrm{ABI}$ in a Taiwanese population. An additive effect between variants of these 2 genes was found. The finding of a potential gene-gene interaction and geneenvironment interaction is interesting, but needs further investigation. (Circ J 2012; 76: 446-452)

Key Words: Ankle-brachial index; BRAP; 9p21; Polymorphisms

$\mathbf{P}$ eripheral artery disease (PAD) may share common genetic and environmental factors with coronary artery disease (CAD) and cerebrovascular disease. ${ }^{1-3}$ PAD is primarily diagnosed by a cutoff value of the anklebrachial index $(\mathrm{ABI})>0.9 .{ }^{4} \mathrm{ABI}$ is not only a diagnostic tool for PAD, but also a predictor of future cardiovascular events. ${ }^{2,3,5-8}$ Although the genetic determinants of PAD remain to be explored, previous studies showed that genes involved in the immune/inflammatory pathway were promising candidate genes for PAD. ${ }^{9}$

Recently, single-nucleotide polymorphisms (SNPs) on the chromosome 9p21 region were found to be significantly associated with myocardial infarction (MI), stroke, CAD and carotid atherosclerosis. ${ }^{10-14}$ The chromosome 9p21 region had also been reported to be associated with PAD in African-Americans and European descents. ${ }^{11,15-17}$ However, there is no report of an association between chromosome 9p21 and PAD in Asian populations. This $9 \mathrm{p} 21$ region contains cyclin-dependent kinase inhibitors $2 \mathrm{~A}$ and $2 \mathrm{~B}$ genes $(C D K N 2 A$ and $C D K N 2 B)$ and the antisense non-coding RNA ( $A N R I L)$ transcribed from the opposite strand of $C D K N 2 A / C D K N 2 B$. Among these 3 genes, $A N R I L$ expression showed the strongest association with cardiovascular phenotypes. ${ }^{10}$ Besides, evidence shows that ANRIL may transcriptionally repress the expression of $C D K N 2 A$ and $C D K N 2 B .^{10,18}$ Therefore, SNPs on ANRIL are more likely to contribute to the susceptibility of the diseases in the previous association studies.

Studies show that $C D K N 2 A$ and $C D K N 2 B$ are abundantly expressed in the atherosclerotic plaques of human coronary arteries. ${ }^{19}$ The 2 genes were involved in cellular senescence and regeneration. Accumulation of senescent cells in the vessel walls could impair regeneration ability and lead to atherosclerotic change. ${ }^{20}$ Furthermore, CDKN2A and CDKN2B may modulate the inflammatory pathway via binding to nuclear

Received August 17, 2011; revised manuscript received October 5, 2011; accepted October 11, 2011; released online November 27,2011 Time for primary review: 19 days

Department of Medical Research (P.-C.T., E.H., Y.-H.Y., S.-H.H.J.), Division of Cardiology (T.-H.L.), Department of Neurology (S.-H.H.J.), Kaohsiung Medical University Hospital, Kaohsiung; Graduate Institute of Medicine (Y.-C.L., E.H.), Department of Internal Medicine (T.-H.L.), Department of Oral Hygiene (Y.-H.Y.), Kaohsiung Medical University, Kaohsiung; Section of Neurology, Taichung Veterans General Hospital, Taichung (Y.-C.L.); and Department of Neurology, National Yang-Ming University School of Medicine, Taipei (Y.-C.L.), Taiwan

Mailing address: Suh-Hang Hank Juo, MD, PhD, Professor, Department of Medical Genetics, Kaohsiung Medical University, 100 TzYou First Road, Kaohsiung City 807, Taiwan, ROC. E-mail: hjuo@kmu.edu.tw

ISSN-1346-9843 doi:10.1253/circj.CJ-11-0925

All rights are reserved to the Japanese Circulation Society. For permissions, please e-mail: cj@j-circ.or.jp 
factor $\kappa-\mathrm{B}(\mathrm{NF}-\kappa \mathrm{B})$ complex. ${ }^{21} \mathrm{We}$ recently found that the pro-inflammatory gene, BRCA-1 associated protein $(B R A P)$, was associated with lower ABI in a Taiwanese population. ${ }^{22}$ Similar to $C D K N 2 A / C N K N 2 B, B R A P$ could modulate the activation of $\mathrm{NF}-\kappa \mathrm{B}$ complex. ${ }^{23}$ Evidence shows that genes involved in the inflammatory pathway might have a synergistic effect on disease susceptibility. ${ }^{24}$ Therefore, the present study tested the individual effect of BRAP and ANRIL on the $\mathrm{ABI}$ value, as well as the combined effects of these 2 genes. To test the effect of these 2 genes, we included our previous $B R A P$ data and genotyped an additional 210 samples in the analyses. Because cigarette smoking is a potent trigger of inflammatory response in the process of atherosclerosis, ${ }^{24,25}$ we also tested the potential gene-environment interaction between BRAP/ANRIL and smoking on atherosclerotic risks.

\section{Methods}

The study protocols and methods were approved by the local Institutional Review Board of Kaohsiung Medical University Hospital. All participants provided written informed consent.

\section{Study Subjects}

A total of 745 asymptomatic, high-risk subjects with a family history of MI or stroke were enrolled from Kaohsiung Medical University Hospital between January 2006 and September 2010. The definition of a high-risk subject is someone who (1) did not have a stroke or MI history at enrollment, and (2) has one first-degree relative or two second-degree relatives who have documented MI or stroke history. Each participant completed a self-administered structured questionnaire that included demographic information, medical history, and medication data. Subjects were classified as non-smokers, former smokers and current smokers. Former smokers were defined as subjects who had quit smoking during the year prior to enrollment. Body mass index (BMI) was calculated as weight $(\mathrm{kg}) /$ height $\left(\mathrm{m}^{2}\right)$. Hypertension was defined as systolic blood pressure (SBP) $\geq 140 \mathrm{mmHg}$ or diastolic blood pressure (DBP) $\geq 90 \mathrm{mmHg}$, a self-reported history of hypertension or taking antihypertensive medications. Diabetes was defined as fasting blood glucose $\geq 126 \mathrm{mg} / \mathrm{dl}$, a self-reported history of diabetes or receiving hypoglycemic medications. Total cholesterol was measured from venous blood after an overnight fast. Hypercholesterolemia was defined as total cholesterol $\geq 200 \mathrm{mg} / \mathrm{dl}$, a self-reported history of hyperlipidemia or taking antihyperlipidemia medications. Among the current study subjects, 535 have been studied in our previous study. ${ }^{22}$

\section{SNP Selection and Genotyping}

Blood was collected for biochemistry analyses and genetic studies. Genomic DNA was isolated from whole blood using the salting-out method, which was standardized with the Puregene kit from Gentra (Research Triangle, NC, USA). SNP rs11066001 for BRAP on chromosome 12q24.12 and 3 SNPs (rs1333040, rs2383207 and rs1333049) for ANRIL on chromosome 9p21.3 were genotyped by the TaqMan method (Applied Biosystems, Foster City, CA, USA). The detailed methods for genotyping were described elsewhere. ${ }^{26}$ The reason why we only selected 1 SNP rs 11066001 for BRAP was because (1) we previously showed that it was associated with $\mathrm{ABI}^{22}$ and $\mathrm{MI}^{23}$ and (2) it is a functional SNP that modulates the transcription efficacy of BRAP. The 3 SNPs on ANRIL were selected from the data of the Hapmap Project (http://hapmap.ncbi.nlm.nih. gov) with a pairwise tagger method. The selection criteria were (1) tagging SNPs covering the whole length of the ANRIL genome (from chr22,073,404 to chr22,115,503) with $\mathrm{r}^{2} \geq 0.8$, (2) SNPs were found to confer risks for $\mathrm{CAD}^{27}$ and carotid atherosclerosis, ${ }^{12,13}$ and (3) SNPs with minor allele frequency (MAF) $\geq 10 \%$ in the Han Chinese population. The genotype call rates of the 4 SNPs ranged from $97.6 \%$ to $99.1 \%$.

\section{Measurement of $\mathrm{ABI}$}

ABI value was measured in resting supine participants using the COLIN VP 1000 (Colin, Komaki, Japan). The validity and reproducibility of the device have been reported. ${ }^{28}$ After entering personal data, blood pressure cuffs were placed on the patients' arms and ankles. Phonocardiography was placed at the second intercostal space over left margin of sternum. Two measurements of blood pressure at bilateral radial and tibial arteries were recorded separately for approximately $1 \mathrm{~min}$, and the mean of 2 measurements were used. The right ABI was calculated directly as the right ankle SBP divided by the largest of the right or left brachial SBP. Similar measures were used for left ABI calculation. The lower ABI value between right and left $\mathrm{ABI}$ data was selected as the final $\mathrm{ABI}$ value for each subject. ${ }^{29}$

\section{Statistical Analysis}

Allele frequency was estimated by direct genotype counting. Hardy-Weinberg equilibrium (HWE) was tested using the $\chi^{2}$ test. Continuous variables are presented as the mean \pm standard deviations (SDs). The relationship between individual cardiovascular risk factors and ABI was evaluated by Pearson's correlation test for continuous variables and Spearman's correlation test for category variables. We used multivariable regression analysis to evaluate the allelic effects of $B R A P$ rs11066001 and ANRIL polymorphisms on ABI with adjustment for traditional cardiovascular risk factors (age, sex, diabetes, hyperlipidemia, hypertension, smoking history and BMI). Multiple testing corrections were performed using the $\max (\mathrm{T})$ permutation procedure for 10,000 times to obtain empirical P value by the PLINK software. ${ }^{30}$ Rs11066001 on $B R A P$ and the most significant SNP on ANRIL were selected in the further analyses of combined genetic effects.

To elucidate the potential gene-gene interaction between $B R A P$ and $A N R I L$, we included an interaction term in the multivariable regression models to test the multiplicative effect. We also computed a gene risk score (GRS) ${ }^{31}$ as a single measure to estimate the additive effect of BRAP and ANRIL on the ABI. The GRS was calculated by counting the number of alleles on the 2 significant SNPs. Each risk allele was assigned to be 1 point. Accordingly, the GRS score for a subject without any risk allele was zero, the score for a subject carrying only one risk allele at either of these 2 SNPs is 1 and the score for a subject carrying 4 risk alleles is 4 . The average ABI on subjects of different GRS were compared by the chisquare trend test. We further stratified the subjects into subgroups by (1) sex or (2) their smoking status. The associations between $\mathrm{ABI}$ and GRS were evaluated by multivariable regression analysis in current smokers, former smokers and non-smokers separately.

We built several predictive models by adding traditional risk factors, SNPs on BRAP or ANRIL, or GRS in the multivariable regression analyses. These predictive models were assessed by correlated Akaike information criterion (AICc) and Schwartz's Bayesian information criterion (BIC), both of which were based on the maximum likelihood estimates of the model parameters. The best model was the 1 with the smallest value of AICc and BIC. The value of $\mathrm{R}^{2}$ was denoted for the proportion of variation that can be explained by the model. 
Table 1. Characteristics of Individuals and the Association With $A B I(n=745)$

\begin{tabular}{lccc} 
& Mean $\pm \mathbf{S D} / \mathbf{n}(\%)$ & $\begin{array}{c}\text { Pearson/Spearman } \\
\text { correlation coefficient }\end{array}$ & P value $^{\dagger}$ \\
Age, years & $54.7 \pm 10.5$ & 0.038 & 0.311 \\
Sex (F) & $407(54.6)$ & 0.229 & $<10^{-4^{*}}$ \\
Hypertension & $280(39.4)$ & 0.157 & $<10^{-4^{*}}$ \\
Diabetes & $98(14.1)$ & 0.047 & 0.212 \\
Hyperlipidemia & $432(62.1)$ & 0.004 & 0.919 \\
BMI & $25.2 \pm 3.8$ & 0.151 & $<10^{-4^{*}}$ \\
Smoker & & 0.104 & $0.006^{*}$ \\
$\quad$ Former & $92(13.2)$ & & \\
$\quad$ Current & $74(10.6)$ & & \\
\hline
\end{tabular}

†The relationship between $\mathrm{ABI}$ and individual risk factors was evaluated by Pearson's correlation test for continuous variables and Spearman's correlation test for category variables.

${ }^{*} \mathrm{P}<0.05$.

$\mathrm{ABI}$, ankle-brachial index; $\mathrm{BMI}$, body mass index.

$\begin{array}{lllll}21,950 & 22,000 & 22,050 & 22,100 & (k b)\end{array}$
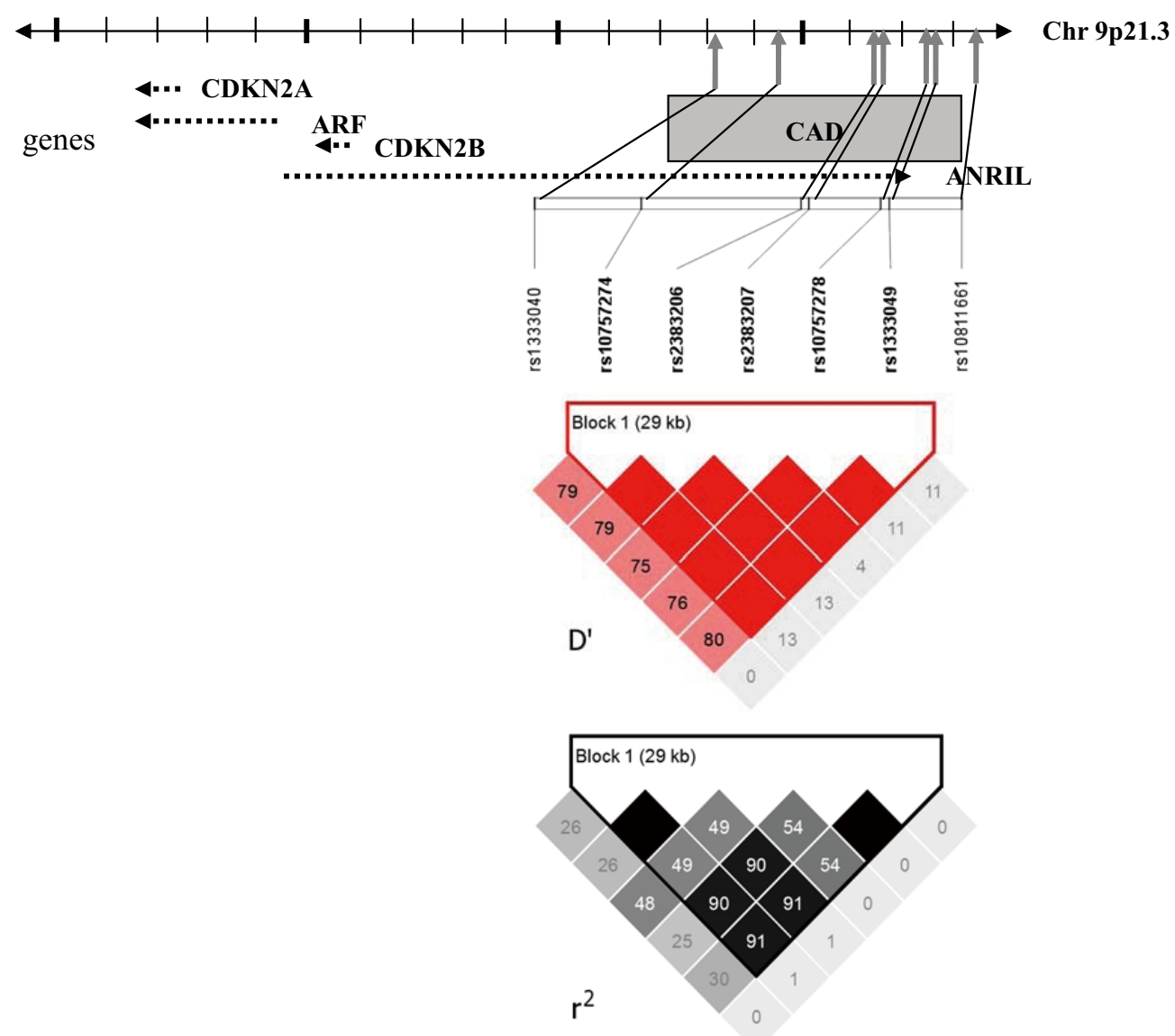

Figure 1. Annotated genes and linkage disequilibrium (LD) plot of 7 SNPs on the chromosome 9p21. The box represents the core risk haplotype for cardiovascular disease (CAD) defined by Broadbent et al. ${ }^{27}$ The LD plot was generated from the data of Hapmap CHB population using Hapview software ( $D^{\prime}$, disequilibrium coefficient; $R^{2}$, correlation coefficient). CDKN2A/2B, cyclindependent kinase inhibitors 2A/2B; ARF, alternate reading frame; ANRIL, antisense non-coding RNA. 


\begin{tabular}{|c|c|c|c|c|c|}
\hline $\begin{array}{l}\text { SNPs } \\
\text { ANRIL }\end{array}$ & \multicolumn{3}{|c|}{$A B I($ mean $\pm S D)$ in each genotype } & $\beta(95 \% \mathrm{Cl})^{\dagger}$ & $\mathbf{P}$ value ${ }^{\dagger}$ \\
\hline \multirow{3}{*}{ rs1333040 } & $C C(n-58)$ & $C T(n-283)$ & $T T(n-361)$ & & \\
\hline & $(n=50)$ & $C(n=<00)$ & $11(n=301)$ & & \\
\hline & $1.113 \pm 0.072$ & $1.104 \pm 0.068$ & $1.096 \pm 0.075$ & $0.007(-0.0004$ to 0.016$)$ & 0.064 \\
\hline \multirow[t]{2}{*}{ rs2383207 } & $\mathrm{AA}(\mathrm{n}=76)$ & $A G(n=303)$ & $G G(n=318)$ & & \\
\hline & $1.115 \pm 0.071$ & $1.105 \pm 0.069$ & $1.094 \pm 0.075$ & $0.010(0.003$ to 0.022$)$ & $0.008^{*}$ \\
\hline \multirow[t]{2}{*}{ rs1333049 } & CC $(n=158)$ & $C G(n=372)$ & $G G(n=173)$ & & \\
\hline & $1.091 \pm 0.083$ & $1.102 \pm 0.067$ & $1.107 \pm 0.077$ & $-0.010(-0.018$ to -0.003$)$ & $0.015^{\star}$ \\
\hline \multicolumn{6}{|l|}{ BRAP } \\
\hline \multirow[t]{2}{*}{ rs11066001 } & GG $(n=58)$ & $\mathrm{GA}(\mathrm{n}=307)$ & $A A(n=373)$ & & \\
\hline & $1.082 \pm 0.080$ & $1.096 \pm 0.070$ & $1.104 \pm 0.075$ & $-0.009(-0.018$ to -0.001$)$ & $0.036^{*}$ \\
\hline
\end{tabular}

tThe additive effects of BRAP rs11066001 and ANRIL polymorphisms on the $\mathrm{ABI}$ were separately evaluated by multivariable regression analysis adjusting for traditional cardiovascular risk factors (age, sex, diabetes, hyperlipidemia, hypertension, smoking history and $\mathrm{BMI}$ ).

${ }^{*}$ Empirical $P$ value $<0.05$.

SNPs, single-nucleotide polymorphisms; $\mathrm{Cl}$, confidence interval. Obbreviations see in Table 1.

Adjusted R2 (Adj_R ${ }^{2}$ ) was used to compare different statistical models with consideration of the number of parameters included in the model. A 2-tailed $\mathrm{P}$ value of $<0.05$ was considered significance. Statistical analyses were performed with JMP software (version 9.0; SAS Institute Inc, Cary, NC, USA).

\section{Results}

A total of 745 participants were included in the present study. The distribution of ABI in our study participants approximates a normal distribution ( $\mathrm{P}$ value for Kolmogorov-Smirnov test $>0.05$; mean \pm SD: $1.10 \pm 0.07$; range: $0.73-1.32$ ). The demographic information, biochemical data and the association with ABI values are shown in Table 1 . The mean age of our study participants was $54.7 \pm 10.5$ years (range: $25-94$ ), and $54.6 \%$ of our subjects were female. Univariate analyses demonstrated that sex, hypertension, BMI and smoking status were significantly associated with ABI (Table 1). In the subsequent analysis of genetic effect, we included all these significant covariates in the multivariable regression analyses.

\section{SNP Data}

Three tagging SNPs on ANRIL (ie, rs1333040, rs2383207 and rs1333049) and rs11066001 on BRAP were genotyped. The genotype distribution of these 4 SNPs was in HWE. Two of the 3 SNPs on ANRIL had strong linkage disequilibrium (LD) with other significant SNPs reported in literatures (Figure 1). The MAF of rs1333040, rs2383207, rs1333049 and rs11066001 were $0.29,0.31,0.49$ and 0.29 , respectively, which were similar to the MAF of $0.31,0.33,0.51$ and 0.29 reported in the Han Chinese populations of the Hapmap website.

\section{Effect of $A N R I L$ and $B R A P$ on $A B I$}

The average $A B I$ value among subjects carrying different genotypes of ANRIL and BRAP SNPs are shown in Table 2. SNP rs2383207 and rs1333049 on ANRIL were significantly associated with ABI values, and the risk allele exerted an additive effect. The risk $\mathrm{G}$ allele of rs 2383207 and the risk $\mathrm{C}$ allele of rs1333049 were associated with lower ABI values. For these 2 significant SNPs, each copy of the risk allele was associated with a reduction of $\mathrm{ABI}$ value by 0.011 (empirical $\mathrm{P}=0.008$ ) for SNP rs 2383207 and by 0.010 (empirical $\mathrm{P}=0.015$ ) for SNP rs1333049. For rs1333040, there was a borderline significance in comparison of the ABI values among sub- jects of different genotypes (empirical $\mathrm{P}=0.064$ ). We previously reported that the functional SNP rs11066001 on BRAP was significantly associated with $A B I$ values in a Taiwanese population. ${ }^{22}$ To evaluate the cumulative effect of ANRIL and $B R A P$, we included our previous $B R A P$ data and genotyped an additional 210 subjects. Similar to our previous findings, $\mathrm{G}$ allele carriers of rs11066001 was associated with lower ABI values (empirical $\mathrm{P}=0.036$ ).

\section{Combined Genetic Effects on ABI}

We used regression analysis to test the multivariable effect between BRAP and ANRIL. In the regression model, both of these genes were independently associated with $\mathrm{ABI}(\mathrm{P}=0.003$ for rs2383207 on ANRIL and $\mathrm{P}=0.014$ for rs 11066001 on $B R A P$ ) (Table S1). However, we did not find a significant result for the interaction term ( $\mathrm{P}$ for interaction=0.621). It suggested that these 2 genes had an additive effect rather than a multiplicative effect on ABI.

We also used GRS as a single measure to estimate the additive effect of BRAP and ANRIL. The most significant SNP rs2383207 on ANRIL and rs11066001 on BRAP were chosen to calculate GRS. The GRS was ranged from 0 (with AA genotypes on both SNPs) to 4 (with GG genotypes on both SNPs). Each copy of G allele at rs11066001 or rs2383207 would contribute a 1-point increment in the GRS. We found a higher GRS was significantly associated with a lower ABI value even after adjusting for age, sex, BMI, diabetes, hyperlipidemia, hypertension and smoking history. Additionally, a dose-response relationship between ABI values and GRS was observed for all subjects (adjusted $\mathrm{P}=0.0001$ ) and for sex-specific analysis (adjusted $\mathrm{P}=0.004$ for men and 0.008 for women, Figure 2A). Similar to our previous study, ${ }^{22}$ women had lower ABI values than men did. For men, the average ABI values were highest for those with GRS of 0 , followed by GRS of 2 , and lowest for men with GRS of $4(1.15 \pm 0.08,1.12 \pm 0.07$, and $1.11 \pm 0.05$, respectively). For women, the average $\mathrm{ABI}$ values for GRS 0,2 , and 4 were 1.09 $\pm 0.07,1.08 \pm 0.07$ and $1.05 \pm 0.07$, respectively. We also found that the combined genetic effect on $\mathrm{ABI}$ was strongest in current smokers, followed by former smokers, and lowest in non-smokers (adjusted $\beta=-0.059$ and $\mathrm{P}=0.007$ for current smokers, $\beta=-0.039$ and $\mathrm{P}=0.047$ for former smokers, $\beta=-0.018$ and $\mathrm{P}=0.007$ for non-smokers; Figure 2B). There was a significant interaction between GRS and smoking in the multivariable regression model (adjusted $\mathrm{P}=0.037$ ). 


\section{A}

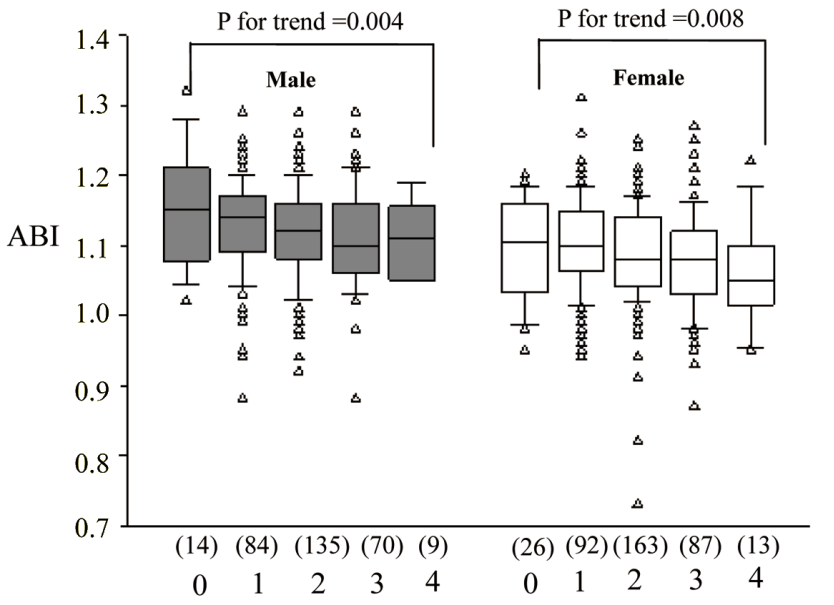

GRS

B

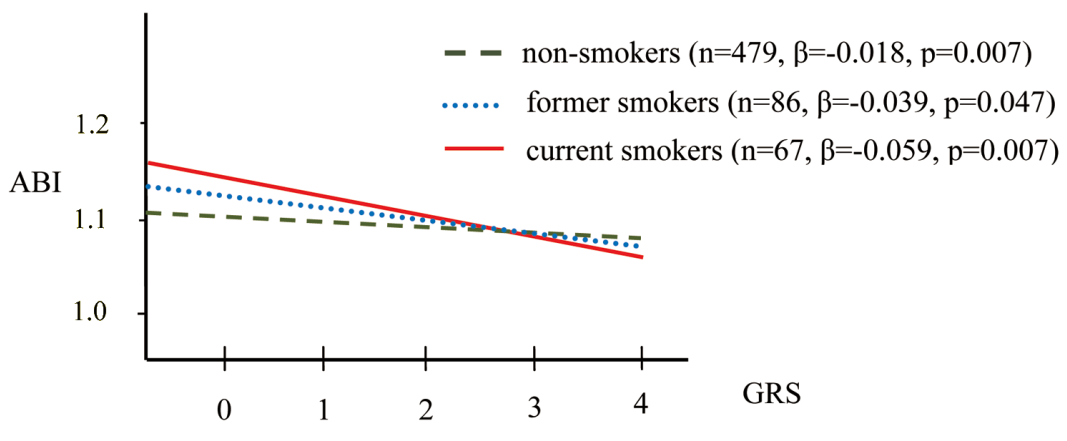

Figure 2. Relationship between the gene risk score (GRS) and ankle-brachial index $(A B I)$ in $(\mathbf{A})$ subjects stratified by sex and (B) subjects stratified by smoking status. All $p$ values were calculated by trend test with adjustment of traditional risk factors and $\beta$ values were used as the slope of line. The number of subjects in each GRS category is shown in parentheses.

\begin{tabular}{|c|c|c|c|}
\hline & $\mathrm{AlCc}^{\dagger}$ & $\mathrm{BIC} \mp$ & $\operatorname{Adj} \_R^{2}(\%)^{\S}$ \\
\hline Model $1^{*}$ & $-1,573.3$ & $-1,533.6$ & 6.6 \\
\hline Model 1+rs11066001 & $-1,577.3$ & $-1,533.2$ & 7.3 \\
\hline Model 1+rs2383207 & $-1,580.2$ & $-1,536.1$ & 7.8 \\
\hline Model $1+$ both SNPs & $-1,584.4$ & $-1,536.0$ & 8.5 \\
\hline Model 1+GRS & $-1,586.4$ & $-1,542.3$ & 8.7 \\
\hline
\end{tabular}

${ }^{*}$ Age, sex, BMI, smoking, diabetes, hypertension and hyperlipidemia.

${ }^{\dagger} \mathrm{AIC} c=-2 \log$ likelihood $+2 k+2 k(k+1) /(n-k-1)$.

$\neq \mathrm{BIC}=-2 \log$ likelihood $+k \ln (n) . k=$ the number of parameters (including intercept and error terms), $n=$ sample size.

$\S A d j \_R^{2}$, adjusted $R^{2}$ by degrees of freedom.

AICc, Akaike information criterion; BIC, Bayesian information criterion. Obbreviations see in Table 1.

We built a series of regression models by adding traditional risk factors, SNPs and GRS. In the multivariable regression model, the traditional risk factors contributed to $6.6 \%$ of ABI variation in our subjects (Table 3). We then added rs11066001 or rs2383207 into the regression model containing traditional risk factors and tested the fitness of different models. The improvement in model fitness was supported by a reduction of AICc and BIC in the new model (Table 3). The model including GRS and traditional risk factors was the best model since it accounted for the highest proportion of $\mathrm{ABI}$ variation $(8.7 \%)$ and had the lowest AICc and BIC (Table 3).

\section{Discussion}

The relationship between lower ABI/PAD and chromosome 9p21 had been reported in other populations, but has not been tested in Asians. In this study, we first reported that rs 2383207 on ANRIL was associated with ABI in a Taiwanese population. The $\mathrm{G}$ allele carriers of rs 2383207 had significantly lower ABI values. We also replicated the association between rs 11066001 on $B R A P$ and ABI. Furthermore, we found that subjects carried risk genotypes at both $B R A P$ rs11066001 and ANRIL rs2383207 had a significantly lower ABI value than other subjects. The combined genetic effect of BRAP and ANRIL was more prominent in smokers than in non-smokers.

ABI was used both as a surrogate for the presence of PAD and for the assessment of risk of cardiovascular diseases. The development of PAD was mediated by genetic determinants and traditional risk factors of atherosclerosis. The conventional risk factors including age, sex, BMI, hyperlipidemia, hypertension, diabetes and cigarette smoking could only explain less than one-fifth of the variation of PAD. ${ }^{32,33}$ The estimated heritability of ABI was higher than $20 \%$ according to the Framingham Offspring cohort. ${ }^{34}$ Genes encoding for interleukin-6 $(I L-6)$, fibrinogen $(F G B)$ and intercellular adhesion molecule-1 (ICAM-1) have been reported to be associated with PAD risks. ${ }^{35}$ The present study showed that both $A N R I L$ and $B R A P$ are risk genes for lower ABI values. In addition to the main effects of $B R A P$ and $A N R I L$, we also demonstrated an additive effect of these 2 genes on ABI.

Although many studies investigated the genetic effect of 9p21 region on CAD, stroke, and MI, only few studies focused 
on its association with PAD. ${ }^{11,15-17}$ We summarized the relevant studies regarding to the associations between SNPs on 9p21 and PAD (Table S2). The Atherosclerosis Risk in Communities (ARIC) Study failed to find an association between rs 10757274 and PAD. ${ }^{16}$ A large meta-analysis combined the data from Iceland, Italy and New Zealand reported a borderline significance between rs10757278 and PAD. ${ }^{11}$ Another SNP rs1333049 was reported to be associated with the PAD in Italians ${ }^{17}$ and Caucasians, ${ }^{15}$ but not in African-Americans. ${ }^{15}$ In coherent to the findings in other ethnic groups, SNP rs 1333049 was associated with ABI in our population. The frequently tested SNPs (rs1330049, rs10757278, rs10757274) in the other ethnic groups and our most significant SNP rs2383207 were located at the same LD block (Figure 1).

For a complex disease, the effect of an individual gene is likely to be small and may be modulated by gene-gene or gene-environment interactions. Using Ingenuity Pathway Analysis (Ingenuity Systems, Redwood City, CA, USA www. ingenuity.com), we found the biological functions of BRAP and 9p21 might be linked by the inflammatory signal pathway (Figure S1). BRAP is a threshold modulator of the Ras-RafMEK-ERK pathway. ${ }^{36}$ When cells are exposed to inflammatory stimuli, the activation of BRAP will initiate a cascade of signal transduction and increase the NF- $\kappa \mathrm{B}$ activity. The Ras signal pathway also affects the transcription of $C D K N 2 A$ and $C D K N 2 B$, and these 2 genes could influence the activity of the $\mathrm{NF}-\kappa \mathrm{B}$ complex. ${ }^{21} \mathrm{NF}-\kappa \mathrm{B}$ is a well-known transcription factor that is able to regulate a number of inflammatory genes. ${ }^{37}$ Therefore, the $9 \mathrm{p} 21$ region and BRAP may both contribute to PAD susceptibility via influencing the NF- $\kappa$ B-related inflammatory pathway. The potential gene-gene interaction between $B R A P$ and 9 p21 warranted further investigation. To note, we found smoking might modulate the combined genetic effect of these 2 genes. Similarly, a community study has reported that the inflammatory gene (IL-6) and smoking have a gene-environmental interaction for atherosclerosis phenotypes. ${ }^{24}$

There are strengths and limitations of the current study. The combined effects between genes involved in the inflammatory pathway were rarely investigated in the context of ABI. Here, we firstly proposed a potential gene-gene interaction between 2 risk genes of ABI. Notably, our statistical result only supported an additive effect between BRAP and 9p21, but failed to find a multiplicative effect. Our data also supported that smoking exposure might enhance the combined genetic effects. Secondly, the relationship between $B R A P$ and $C D K N 2 A / C D K N 2 B$ on $9 \mathrm{p} 21$ was predicted by bioinformatics annotation. Molecular studies are needed to clarify the mechanisms underlying gene-gene interaction between $B R A P$ and $9 \mathrm{p} 21$. In this study, we selected asymptomatic subjects with a family history of MI and stroke as our study population because they were high-risk persons predisposed by genetic factors. We acknowledged that our data may not directly support an association between these 2 genes and PAD because there were only 6 subjects fulfilling the diagnosis of PAD $(\mathrm{ABI}<0.9)$. However, a previous study had shown that a borderline ABI value (0.9-1.0) or a low-normal ABI value (1.01.1) was associated with an increased risk of carotid atherosclerosis. ${ }^{6}$ Because ABI value could be the predictor of both $\mathrm{PAD}$ and other cardiovascular diseases, $, 2,7,8$ our study supported a pro-atherogenic effect of ANRIL and BRAP.

In conclusion, we are the first to report an association between SNP rs2383207 on ANRIL and ABI in the Taiwanese population. Concordant with our previous report, rs11066001 on $B R A P$ is a risk factor for lower ABI. We demonstrated an additive effect between variants on ANRIL and on BRAP. The finding of a potential gene-gene interaction and gene-environment interaction is interesting but needs further investigation.

\section{Acknowledgments}

This work was supported by grants from the following organizations: National Health Research Institutes, Taiwan (NHRI-Ex96-9607PI), National Science Council, Taiwan (NSC 99-2628-B-037-037-MY3 and NSC-99-2320-B-110-001-MY3), Kaohsiung Medical University intramural grant (KMUH98-8I11) and Department of Health, Taiwan (DOH99TD-I-111-TM023).

The authors thank the help from the Statistical Analysis Laboratory, Department of Medical Research, Kaohsiung Medical University Hospital.

Disclosures

Conflict of Interest: none declared.

\section{References}

1. Criqui MH, Langer RD, Fronek A, Feigelson HS, Klauber MR, McCann TJ, et al. Mortality over a period of 10 years in patients with peripheral arterial disease. N Engl J Med 1992; 326: 381-386.

2. Newman AB, Shemanski L, Manolio TA, Cushman M, Mittelmark M, Polak JF, et al. Ankle-arm index as a predictor of cardiovascular disease and mortality in the cardiovascular health study. Arterioscler Thromb Vasc Biol 1999; 19: 538-545.

3. Zheng L, Li J, Hu D, Luo Y, Li X, Xu Y, et al. Association of low ankle-brachial index with mortality in patients with ischemic heart disease. J Atheroscler Thromb 2010; 17: 759-767.

4. Hirsch AT, Haskal ZJ, Hertzer NR, Bakal CW, Creager MA, Halperin JL, et al. ACC/AHA guidelines for the management of patients with peripheral arterial disease (lower extremity, renal, mesenteric, and abdominal aortic). Circulation 2006; 113: 463-465.

5. Igarashi Y, Chikamori T, Hida S, Tanaka H, Shiba C, Usui Y, et al. Importance of the ankle-brachial pressure index in the diagnosis of coronary artery disease in women with diabetes without anginal pain. Circ J 2011; 75: 2206-2212.

6. McDermott MM, Liu K, Criqui MH, Ruth K, Goff D, Saad MF, et al. Ankle-brachial index and subclinical cardiac and carotid disease: The multi-ethnic study of atherosclerosis. Am J Epidemiol 2005; 162: 33-41.

7. Busch MA, Lutz K, Rohl JE, Neuner B, Masuhr F. Low anklebrachial index predicts cardiovascular risk after acute ischemic stroke or transient ischemic attack. Stroke 2009; 40: 3700-3705.

8. Wild SH, Byrne CD, Smith FB, Lee AJ, Fowkes FG. Low anklebrachial pressure index predicts increased risk of cardiovascular disease independent of the metabolic syndrome and conventional cardiovascular risk factors in the edinburgh artery study. Diabetes Care 2006; 29: 637-642.

9. Fu S, Zhao H, Shi J, Abzhanov A, Crawford K, Ohno-Machado L, et al. Peripheral arterial occlusive disease: Global gene expression analyses suggest a major role for immune and inflammatory responses. BMC Genomics 2008; 9: 369.

10. Cunnington MS, Santibanez Koref M, Mayosi BM, Burn J, Keavney B. Chromosome 9p21 SNPs associated with multiple disease phenotypes correlate with anril expression. PLoS Genet 2010; 6: e1000899.

11. Helgadottir A, Thorleifsson G, Magnusson KP, Gretarsdottir S, Steinthorsdottir V, Manolescu A, et al. The same sequence variant on 9p21 associates with myocardial infarction, abdominal aortic aneurysm and intracranial aneurysm. Nat Genet 2008; 40: 217-224.

12. Lin HF, Tsai PC, Liao YC, Lin TH, Tai CT, Juo SH, et al. Chromosome 9p21 genetic variants are associated with myocardial infarction but not with ischemic stroke in a Taiwanese population. J Invest Med 2011; 59: 926-930.

13. Lin HF, Tsai PC, Lin RT, Khor GT, Sheu SH, Juo SH. Sex differential genetic effect of chromosome 9 p21 on subclinical atherosclerosis. PLoS One 2010; 5: e15124.

14. McPherson R, Pertsemlidis A, Kavaslar N, Stewart A, Roberts R, Cox DR, et al. A common allele on chromosome 9 associated with coronary heart disease. Science 2007; 316: 1488-1491.

15. Cluett C, McDermott MM, Guralnik J, Ferrucci L, Bandinelli S, Miljkovic I, et al. The 9p21 myocardial infarction risk allele increases risk of peripheral artery disease in older people. Circ Cardiovasc Genet 2009; 2: 347-353.

16. Yamagishi K, Folsom AR, Rosamond WD, Boerwinkle E. A genetic variant on chromosome $9 \mathrm{p} 21$ and incident heart failure in the aric study. Eur Heart J 2009; 30: 1222-1228.

17. Ye S, Willeit J, Kronenberg F, Xu Q, Kiechl S. Association of genetic 
variation on chromosome 9p21 with susceptibility and progression of atherosclerosis: A population-based, prospective study. J Am Coll Cardiol 2008; 52: 378-384.

18. Pasmant E, Sabbagh A, Vidaud M, Bieche I. ANRIL, a long, noncoding RNA, is an unexpected major hotspot in GWAS. FASEB J 2010; 25: 444-448

19. Holdt LM, Sass K, Gabel G, Bergert H, Thiery J, Teupser D. Expression of chr9p21 genes CDKN2B (p15(INK4B)), CDKN2A (p16(INK4A), $\mathrm{p} 14(\mathrm{ARF}))$ and MTAP in human atherosclerotic plaque. Atherosclerosis 2010; 214: $264-270$.

20. Minamino T, Komuro I. Vascular cell senescence: Contribution to atherosclerosis. Circ Res 2007; 100: 15-26.

21. Rocha S, Campbell KJ, Perkins ND. P53- and MDM2-independent repression of NF-kappa B transactivation by the ARF tumor suppressor. Mol Cell 2003; 12: $15-25$.

22. Tsai PC, Lin TH, Hsu PC, Wang YS, Lia YC, Juo SH. A polymorphism of $270 \mathrm{~A}>\mathrm{G}$ in BRAP is associated with lower ankle-brachial index in a Taiwanese population. J Atheroscler Thromb 2011; 18: $413-420$.

23. Ozaki K, Sato H, Inoue K, Tsunoda T, Sakata Y, Mizuno H, et al. SNPs in BRAP associated with risk of myocardial infarction in Asian populations. Nat Genet 2009; 41: 329-333.

24. Jerrard-Dunne P, Sitzer M, Risley P, Buehler A, von Kegler S, Markus HS. Inflammatory gene load is associated with enhanced inflammation and early carotid atherosclerosis in smokers. Stroke 2004; 35: $2438-2443$

25. Kubozono T, Miyata M, Ueyama K, Hamasaki S, Kusano K, Kubozono $\mathrm{O}$, et al. Acute and chronic effects of smoking on arterial stiffness. Circ J 2011; 75: 698-702.

26. Hsiao PJ, Lu MY, Chiang FY, Shin SJ, Tai YD, Juo SH. Vascular endothelial growth factor gene polymorphisms in thyroid cancer. $J$ Endocrinol 2007; 195: 265-270.

27. Broadbent HM, Peden JF, Lorkowski S, Goel A, Ongen H, Green F et al. Susceptibility to coronary artery disease and diabetes is encoded by distinct, tightly linked SNPs in the ANRIL locus on chromosome 9p. Hum Mol Genet 2008; 17: 806-814.

28. Yamashina A, Tomiyama H, Takeda K, Tsuda H, Arai T, Hirose K, et al. Validity, reproducibility, and clinical significance of noninvasive brachial-ankle pulse wave velocity measurement. Hypertens Res 2002; 25: 359-364.

29. Allison MA, Aboyans V, Granston T, McDermott MM, Kamineni $\mathrm{A}, \mathrm{Ni} \mathrm{H}$, et al. The relevance of different methods of calculating the ankle-brachial index: The multi-ethnic study of atherosclerosis. Am J Epidemiol 2010; 171: 368-376.

30. Purcell S, Neale B, Todd-Brown K, Thomas L, Ferreira MA, Bender $\mathrm{D}$, et al. Plink: A tool set for whole-genome association and population-based linkage analyses. Am J Hum Genet 2007; 81: 559-575.

31. Liao YC, Lin HF, Rundek T, Cheng R, Guo YC, Sacco RL, et al. Segment-specific genetic effects on carotid intima-media thickness: The northern manhattan study. Stroke 2008; 39: 3159-3165.

32. Lee HY, Oh BH. Aging and arterial stiffness. Circ J 2010; 74: $2257-$ 2262.

33. Kullo IJ, Bailey KR, Kardia SL, Mosley TH Jr, Boerwinkle E, Turner ST. Ethnic differences in peripheral arterial disease in the nhlbi genetic epidemiology network of arteriopathy (genoa) study. Vasc Med 2003; 8: 237-242.

34. Murabito JM, Guo CY, Fox CS, D'Agostino RB. Heritability of the ankle-brachial index: The Framingham Offspring study. Am J Epidemiol 2006; 164: $963-968$.

35. Knowles JW, Assimes TL, Li J, Quertermous T, Cooke JP. Genetic susceptibility to peripheral arterial disease: A dark corner in vascular biology. Arterioscler Thromb Vasc Biol 2007; 27: 2068-2078.

36. Matheny SA, Chen C, Kortum RL, Razidlo GL, Lewis RE, White MA Ras regulates assembly of mitogenic signalling complexes through the effector protein imp. Nature 2004; 427: 256-260.

37. Reich LM, Heiss G, Boland LL, Hirsch AT, Wu K, Folsom AR. Ankle-brachial index and hemostatic markers in the Atherosclerosis Risk in Communities (ARIC) study cohort. Vasc Med 2007; 12: 267 273.

\section{Supplemental Files}

Supplemental Files 1

Table S1. Multivariable Regression Analysis to Evaluate the Multiplicative Effect Between ANRIL and BRAP on ABI

Table S2. Relevant Studies Regarding the Associations of Polymorphisms on 9p21 and ABI and PAD

Figure S1. The protein-protein interaction between BRAP and 9p21 by IPA.

Please find supplemental file(s);

http://dx.doi.org/10.1253/circj.CJ-11-0925 